\title{
Spin-Peierls transition in TiOCl
}

\author{
Mohammad Shaz, ${ }^{1}$ Sander van Smaalen, ${ }^{1, *}$ Lukas Palatinus, ${ }^{1,2}$ Markus Hoinkis, ${ }^{3}$ Matthias Klemm, ${ }^{3}$ Siegfried Horn, ${ }^{3}$ and \\ Ralph Claessen ${ }^{3}$ \\ ${ }^{1}$ Laboratory of Crystallography, University of Bayreuth, 95440 Bayreuth, Germany \\ ${ }^{2}$ Institute of Physics of the Academy of Sciences of the Czech Republic, Prague, Czech Republic \\ ${ }^{3}$ Experimentalphysik II, University of Augsburg, D-86135 Augsburg, Germany
}

(Received 18 January 2005; published 11 March 2005)

\begin{abstract}
Temperature-dependent $\mathrm{x}$-ray diffraction of the low-dimensional spin-1/2 quantum magnet TiOCl shows that the phase transition at $T_{c 2}=90 \mathrm{~K}$ corresponds to a lowering of the lattice symmetry. Below $T_{c 1}=66 \mathrm{~K}$ a twofold superstructure develops, that indicates the formation of spin-singlet pairs via direct exchange between neighboring Ti atoms, while the role of superexchange is found to be negligible. TiOCl thus is identified as a spin-Peierls system of pure one-dimensional chains of atoms. The first-order character of the transition at $T_{c 1}$ is explained by the competition between the structurally deformed state below $T_{c 2}$ and the spin-Peierls state below $T_{c 1}$.
\end{abstract}

DOI: 10.1103/PhysRevB.71.100405

PACS number(s): 75.30.Et, 75.30.Kz, 61.50.Ks, 61.66.Fn

Low-dimensional $S=1 / 2$ quantum spin systems are of interest, because of the importance for understanding the mechanism of high- $T_{c}$ superconductivity, ${ }^{1}$ as well as for their potential applications in quantum computers. Furthermore, the relatively simple, yet complicated materials with onedimensional (1D) or two-dimensional (2D) quantum spin systems offer a wide variety of ground states, that are accessible by $a b$ initio theory, and therefore might help towards the understanding of fundamental quantum-mechanical properties of solids. The development of magnetic order at low temperatures may or may not be coupled to a change of the electronic structure, resulting in ground states with antiferromagnetic order or spin-density waves (SDW). The spinPeierls state is defined by singlet pairs of localized electrons that form because of an enhancement of exchange interactions between neighboring magnetic atoms due to a dimerization of the crystal structure.

$\mathrm{CuGeO}_{3}$ is the only inorganic compound for which the spin-Peierls state below $T_{c}=14 \mathrm{~K}$ has been unambiguously established. ${ }^{2-5}$ Initially $\mathrm{NaV}_{2} \mathrm{O}_{5}$ was considered to be a candidate spin-Peierls material, but more recent work showed that the fourfold superstructure below $T_{c}=34 \mathrm{~K}$ is related to a combination of charge-, orbital-, and magnetic order. ${ }^{6} \mathrm{Re}-$ cently, Seidel et al. $^{7}$ proposed that TiOCl is a 1D $S=1 / 2$ quantum spin system that transforms into a spin-Peierls state at low temperatures.

$\mathrm{TiOCl}$ crystallizes in a layered structure ${ }^{8}$ (Fig. 1) in which two different types of chains of $\mathrm{Ti}^{3+}, d^{1}(S=1 / 2)$, have been identified. ${ }^{7}$ The chain along a allows interactions between the electrons via superexchange, whereas the chain along $\mathbf{b}$ supports direct exchange interactions. The latter type of chains has been proposed to be responsible for the quasi-1D character of the magnetic interactions, as evidenced by the magnetic susceptibility, electron-spin resonance (ESR), IR reflectivity, angle-resolved photoelectron spectroscopy (ARPES), and electronic band-structure calculations., ${ }^{79-12}$ Based on the temperature dependencies of the magnetic susceptibility (Ref. 7), ESR (Ref. 9), and NMR (Ref. 13), a second-order phase transition was found at $T_{c 2}=94 \mathrm{~K}$, while a first-order transition takes place at $T_{c 1}=67 \mathrm{~K}$. The latter transition corresponds to a sudden development of magnetic order, accompanied by a doubling of the lattice constant along b (Refs. 7 and 13). The magnetic moments are zero below $T_{c 1}$, and the size $\left(E_{g}=430 \mathrm{~K}\right)$ of the spin gap has been

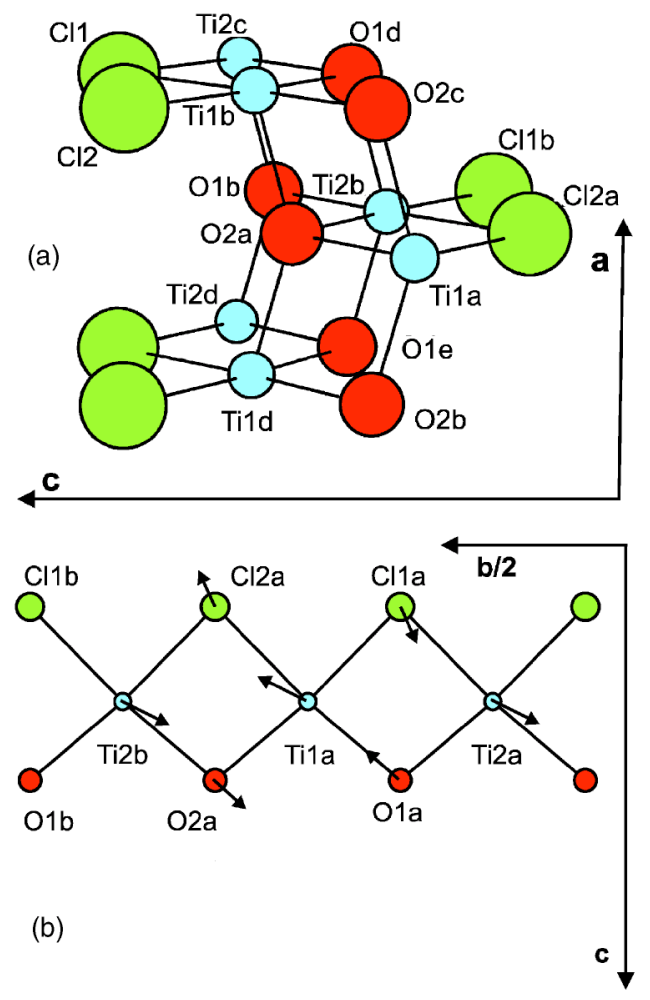

FIG. 1. (Color online) Crystal structure of TiOCl. (a) Perspective view of one layer. (b) The ribbon parallel to $\mathbf{b}$ at $x=0$, containing a chain of $\mathrm{Ti}$ atoms. The displacements in the superstructure are given by arrows $(20 \times$ the true values). Crystallographically independent atoms are indicated by numbers. Different but symmetry equivalent atoms are indicated by an additional letter that corresponds to Table I. The unit-cell axes are indicated. (Blue for Ti, red for $\mathrm{O}$, and green for $\mathrm{Cl}$.) 
taken as an indication for a nonconventional spin-Peierls state at low temperatures. ${ }^{13}$ Above $T_{c 2}$ up to $T^{*}=135 \mathrm{~K}$ a pseudo-spin-gap due to fluctuations has been found. ${ }^{10,13}$

In the present contribution we report the discovery of superlattice reflections in the x-ray diffraction of TiOCl below $T_{c 1}=66 \mathrm{~K}$ as well as a complete structure determination at $T=10 \mathrm{~K}$. The latter shows that the twofold superstructure can be interpreted as a dimerization of the $1 \mathrm{D}$ chains of $\mathrm{Ti}$ atoms along $\mathbf{b}$, while the interatomic distances and bond angles between chains are much less affected by the structural deformation. Our results thus indicate that, despite the unconventional magnetic behavior observed at higher temperatures, the opening of the spin gap below $66 \mathrm{~K}$ is due to a spin-Peierls transition, where a surprisingly simple dimerization of the 1D Ti atom chains is responsible for the formation of spin singlets.

Single crystals of $\mathrm{TiOCl}$ were prepared by gas transport in evacuated quartz glass tubes, following published procedures. ${ }^{8}$ Single-crystal $\mathrm{x}$-ray diffraction with synchrotron radiation was performed at beamline D3 of Hasylab (DESY, Hamburg), employing monochromatized radiation of wavelength $0.5000 \AA$. A single crystal of dimensions $0.05 \times 0.11 \times 0.01 \mathrm{~mm}^{3}$ was mounted on a carbon fiber attached to a closed-cycle helium cryostat mounted on a Huber four-circle diffractometer. X-ray diffraction was measured by a point detector.

In a first experiment $q$ scans were performed at $T=10 \mathrm{~K}$ along the reciprocal lattice lines $(h+\xi, k, l),(h, k+\xi, l)$, $(h+1 / 2, k+\xi, l),(h+1 / 2, k+\xi, l+1 / 2)$, and $(h, k+\xi, l+1 / 2)$ for approximately 20 different $(h, k, l)$ combinations, and with $\xi$ scanned from 0 to 1 . Superlattice reflections were only found at $(h, k+1 / 2, l)$ positions, indicating a doubling of the unit cell along b [Fig. 2(a)]. A few of the strongest superlattice reflections were selected for temperature-dependent measurements. $q$ scans established that the relative positions of the superlattice reflections are independent of temperature. The integrated intensities were measured by $\omega$ scans. Up to about $65 \mathrm{~K}$, they were found to be independent of temperature, but then they dropped continuously to zero at $67 \mathrm{~K}$ [Fig. 2(b)], which can be explained by the coexistence of the intermediate- and low-temperature phases, as can be the result of internal strains within the sample. $q$ scans above $67 \mathrm{~K}$ did not give any evidence for an incommensurate superstructure. Scans of several main reflections showed that for $T$ $\leqslant 90 \mathrm{~K}$ their full width at half maximum (FWHM) is larger than for $T \geqslant 91 \mathrm{~K}$ (Fig. 3). The broadening was reversible and gradually increased on decreasing temperature, but it did not show a clear anomaly at $T_{c 1}$. The splitting of reflections was not resolved, thus preventing the determination of the space group of the intermediate phase.

These results indicate a second-order phase transition at $T_{c 2}=90(1) \mathrm{K}$ corresponding to a lowering of the point symmetry of the crystal structure, followed by a first-order phase transition at $T_{c 1}=66(1) \mathrm{K}$ towards a structure with a doubled b axis. The $q$ scans at $67 \mathrm{~K}$ show that the transition at $T_{c 1}$ does not correspond to an incommensuration of the superstructure, contrary to the suggestion by NMR (Ref. 13). Alternatively, the reduced lattice symmetry below $T_{c 2}$ offers an explanation for the broad resonances in NMR.
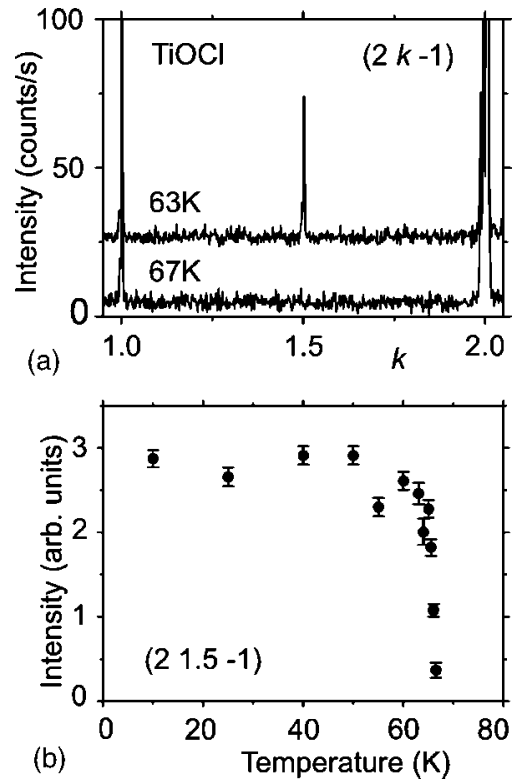

FIG. 2. Superlattice reflections of TiOCl below $T_{c 1}=66 \mathrm{~K}$. (a) $q$ scan $(2,1+v,-1)$ with $-0.05<v<1.05$ at temperatures of $63 \mathrm{~K}$ (shifted by 30 counts/s) and $67 \mathrm{~K}$. (b) Temperature dependence of the integrated intensities of the superlattice reflection $(2,1.5,-1)$. The intensity at $T=67 \mathrm{~K}$ was measured as less than zero (zero within standard uncertainty). The error bars indicate standard uncertainties.

At $T=10 \mathrm{k}$ we have measured the integrated intensities of all Bragg reflections up to $[\sin (\theta) / \lambda]_{\max }=0.7 \AA^{-1}$. Structure refinements were performed within the superspace approach applied to this commensurately modulated structure, ${ }^{14,15}$ with the superspace group $\operatorname{Pmmn}(0,0.5,0)$ and with lattice parameters $a=3.7829, \quad b_{0}=3.3415, \quad$ and $c=8.0305 \AA$ at $T=10 \mathrm{~K}$. Different sections $t_{0}$ of superspace correspond to superstructures $a \times 2 b_{0} \times c$ with different symmetries. ${ }^{15} \mathrm{Un}$ der the assumption of twinning, the best fit to the diffraction data was obtained for $t_{0}=0.125$, corresponding to a supercell with monoclinic symmetry and space group $P 2_{1} / \mathrm{m}$ (a axis unique). The fit with $t_{0}=0.0$ was nearly as good. It corre-

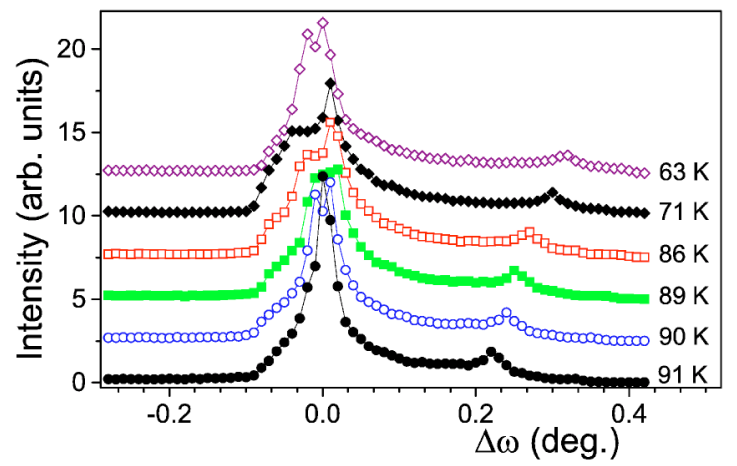

FIG. 3. (Color online) $\omega$ scans of the $(0,2,0)$ main reflection at several temperatures. Scans at $T>91 \mathrm{~K}$ (data not shown) exhibit similar widths as the scan at $91 \mathrm{~K}$. Each scan is centered on $\Delta \omega=0$, and an incremental offset of 2.5 has been applied. The large width near the base of the reflections and the secondary maximum are the result of the relatively poor quality of the single crystal. 
TABLE I. Selected interatomic distances and bond angles in the superstructure of TiOCl at $10 \mathrm{~K}$. Crystallographically independent atoms are indicated by numbers 1 and 2. Different but symmetry equivalent atoms are indicated by an additional letter. Standard uncertainties are given in parentheses.

\begin{tabular}{|c|c|c|c|}
\hline Atoms & $\begin{array}{c}\text { Distance } \\
(\AA)\end{array}$ & Atoms & $\begin{array}{c}\text { Distance } \\
(\AA)\end{array}$ \\
\hline Tila $\mathrm{a}-\mathrm{Ti} 2 \mathrm{a}^{\mathrm{a}}$ & $3.429(1)$ & Tila-Tilb $b^{b}$ & $3.159(1)$ \\
\hline Ti1 $a-T i 2 b^{a}$ & $3.254(1)$ & $\mathrm{Ti} 1 \mathrm{~b}-\mathrm{Ti} 2 \mathrm{~b}^{\mathrm{b}}$ & $3.177(1)$ \\
\hline $\mathrm{Tilb}-\mathrm{Ti}^{\mathrm{d}} \mathrm{d}^{\mathrm{c}}$ & 3.783 & $\mathrm{Ti} 2 \mathrm{~b}-\mathrm{Ti} 2 \mathrm{c}^{\mathrm{b}}$ & $3.198(1)$ \\
\hline $\mathrm{Ti} 2 \mathrm{c}-\mathrm{Ti} 2 \mathrm{~d}^{\mathrm{c}}$ & 3.783 & & \\
\hline Ti1a $-01 a^{a}$ & $2.203(3)$ & $\mathrm{Ti} 2 \mathrm{~b}-\mathrm{O} 1 \mathrm{~b}^{\mathrm{a}}$ & $2.222(3)$ \\
\hline Tila $-O 2 a^{a}$ & $2.173(4)$ & $\mathrm{Ti} 2 \mathrm{~b}-\mathrm{O} 2 \mathrm{a}^{\mathrm{a}}$ & $2.189(3)$ \\
\hline $\mathrm{Ti} 1 \mathrm{a}-\mathrm{O} 2 \mathrm{~b}^{\mathrm{b}}$ & $1.956(1)$ & $\mathrm{Ti} 2 \mathrm{~b}-\mathrm{O} 1 \mathrm{~d}^{\mathrm{b}}$ & $1.959(1)$ \\
\hline Ti1a-O2 $c^{b}$ & $1.956(1)$ & $\mathrm{Ti} 2 \mathrm{~b}-\mathrm{O} 1 \mathrm{e}^{\mathrm{b}}$ & $1.959(1)$ \\
\hline Tila $-C 11 a^{a}$ & $2.398(2)$ & $\mathrm{Ti} 2 \mathrm{~b}-\mathrm{Cl} \mathrm{b}^{\mathrm{a}}$ & $2.407(2)$ \\
\hline Tila $-\mathrm{Cl} 2 \mathrm{a}^{\mathrm{a}}$ & $2.383(2)$ & $\mathrm{Ti} 2 \mathrm{~b}-\mathrm{Cl} 2 \mathrm{a}^{\mathrm{a}}$ & $2.394(2)$ \\
\hline Atoms & $\begin{array}{l}\text { Angle } \\
\text { (deg.) }\end{array}$ & Atoms & $\begin{array}{l}\text { Angle } \\
\text { (deg.) }\end{array}$ \\
\hline Ti1a-O1a-Ti2a $a^{a}$ & $101.6(2)$ & Ti1a - Cl1a - Ti2 $a^{a}$ & $91.1(1)$ \\
\hline Ti1a-O2a-Ti2b $b^{a}$ & $96.5(2)$ & Ti1a $-\mathrm{Cl} 2 \mathrm{a}-\mathrm{Ti}_{2} \mathrm{~b}^{\mathrm{a}}$ & $85.9(1)$ \\
\hline Tila $-\mathrm{O} 2 \mathrm{a}-\mathrm{Ti}^{\mathrm{b}} \mathrm{b}^{\mathrm{b}}$ & $99.7(1)$ & $\mathrm{Ti} 1 \mathrm{~b}-\mathrm{O} 1 \mathrm{~d}-\mathrm{Ti} 2 \mathrm{~b}^{\mathrm{b}}$ & $99.4(1)$ \\
\hline $\mathrm{Ti} 2 \mathrm{~b}-\mathrm{O} 1 \mathrm{~b}-\mathrm{Ti} 2 \mathrm{c}^{\mathrm{b}}$ & $99.6(1)$ & $\mathrm{Ti} 1 \mathrm{~b}-\mathrm{O} 2 \mathrm{a}-\mathrm{Ti} 2 \mathrm{~b}^{\mathrm{b}}$ & $99.9(1)$ \\
\hline $\mathrm{Ti} 1 \mathrm{~b}-\mathrm{O} 2 \mathrm{a}-\mathrm{Ti}^{\mathrm{c}} \mathrm{d}^{\mathrm{c}}$ & $150.4(2)$ & $\mathrm{Ti} 2 \mathrm{c}-\mathrm{O} 1 \mathrm{~b}-\mathrm{Ti} 2 \mathrm{~d}^{\mathrm{c}}$ & $149.8(2)$ \\
\hline
\end{tabular}

${ }^{\mathrm{a}}$ Distances and angles within a single ribbon parallel to $\mathbf{b}$.

${ }^{b}$ Distances and angles between neighboring ribbons, $\mathbf{a} / 2$ apart.

${ }^{c}$ Distances and angles towards ribbons at $\pm \mathbf{a}$.

sponds to a supercell with acentric orthorhombic symmetry Pmm2 (Ref. 17). The two structure models are completely different. Pmm 2 involves three crystallographically independent $\mathrm{Ti}$ and $\mathrm{Cl}$ atoms that are distributed over two independent ribbons along b. $P 2_{1} / m$ involves two independent $\mathrm{Ti}$ and $\mathrm{Cl}$ atoms that alternate along a single unique ribbon along b (Fig. 1). NMR indicates two crystallographically independent $\mathrm{Ti}$ and $\mathrm{Cl}$ atoms at low temperatures. ${ }^{13}$ It can therefore be concluded that the superlattice structure has monoclinic symmetry $P 2_{1} / \mathrm{m}$.

One ribbon parallel to $\mathbf{b}$ contains the six crystallographically independent atoms in the supercell $P 2_{1} / m$, all of which are in the mirror plane. Accordingly, the displacements of the atoms are restricted to the b,c plane [Fig. 1(b)]. The pattern of displacements clearly shows a dimerization of the chain of $\mathrm{Ti}$ atoms, while the displacements of $\mathrm{O}$ and $\mathrm{Cl}$ atoms are such as to minimize the elastic strain of the structure. This interpretation is supported by comparing the interatomic distances in the superstructure (Table I) and the basic structure (Table II). The largest variation of $0.18 \AA$ is found for the alternating distances on the $\mathrm{Ti}$ chains along $\mathbf{b}$, indicating the formation of $\mathrm{Ti}-\mathrm{Ti}$ pairs on these chains. The shortest $\mathrm{Ti}-\mathrm{Ti}$ distance is between chains, but its variation in the superstructure is much less than the variation of distances along the chains, thus supporting the model of pair building on the chains as opposed to the formation of singlet pairs between electrons on neighboring chains. The smallest variation $(0.024 \AA)$ is obtained for the four independent $\mathrm{Ti}-\mathrm{Cl}$ distances, while the variation of $\mathrm{Ti}-\mathrm{O}$ distances $(0.049 \AA)$ is still much smaller than the variation of $\mathrm{Ti}-\mathrm{Ti}$ distances along the chains. This interpretation of the superstructure supports the model of spin pairing on chains of $\mathrm{Ti}$ atoms along b via direct exchange. ${ }^{7}$ Although bond angles $\mathrm{Ti}-\mathrm{O}-\mathrm{Ti}$ and $\mathrm{Ti}-\mathrm{Cl}-\mathrm{Ti}$ alternate along the chains, they do not provide evidence for a role of superexchange in the spin-Peierls transition.

The crystal structure at room temperature and the basic

TABLE II. Distances ( $\AA$ ) and bond angles (deg.) in the basic structures of $\mathrm{TiOCl}$ at room temperature and at $T=10 \mathrm{~K}$. One crystallographically independent atom each exists for $\mathrm{Ti}, \mathrm{O}$, and $\mathrm{Cl}$.

\begin{tabular}{lcc}
\hline \hline \multicolumn{1}{c}{ Atoms } & $T=10 \mathrm{~K}$ & $T=295 \mathrm{~K}$ \\
\hline $\mathrm{Ti}-\mathrm{Ti}^{\mathrm{a}}$ & 3.342 & 3.355 \\
$\mathrm{Ti}-\mathrm{O}^{\mathrm{a}}$ & $2.196(1)$ & $2.187(2)$ \\
$\mathrm{Ti}-\mathrm{Cl}^{\mathrm{a}}$ & $2.395(1)$ & $2.393(1)$ \\
$\mathrm{Ti}-\mathrm{O}-\mathrm{Ti}^{\mathrm{a}}$ & $99.2(1)$ & $100.2(1)$ \\
$\mathrm{Ti}-\mathrm{Cl}-\mathrm{Ti}^{\mathrm{a}}$ & $88.4(1)$ & $89.0(1)$ \\
$\mathrm{Ti}-\mathrm{Ti}$ & $3.177(1)$ & $3.172(1)$ \\
$\mathrm{Ti}-\mathrm{O}^{\mathrm{b}}$ & $1.958(1)$ & $1.958(1)$ \\
$\mathrm{Ti}-\mathrm{O}-\mathrm{Ti}^{\mathrm{b}}$ & $99.7(1)$ & $99.7(1)$ \\
$\mathrm{Ti}-\mathrm{Ti}^{\mathrm{c}}$ & 3.783 & 3.779 \\
$\mathrm{Ti}-\mathrm{O}-\mathrm{Ti}^{\mathrm{c}}$ & $150.0(1)$ & $149.5(2)$ \\
\hline \hline
\end{tabular}

${ }^{a}$ Distances and angles within a single ribbon parallel to $\mathbf{b}$.

${ }^{b}$ Distances and angles between neighboring ribbons, $\mathbf{a} / 2$ apart.

${ }^{\mathrm{c}}$ Distances and angles towards ribbons at $\pm \mathbf{a}$. 
structure at $10 \mathrm{~K}$ are nearly equal, with the small differences in interatomic distances explained by thermal expansion (Table II). This implies that structural changes within Pmmn cannot be at the origin of the observed anomalies at $T^{*}$. Instead the anomalous behavior in the temperature dependencies of the magnetic susceptibility and spectroscopic properties below $T^{*}=135 \mathrm{~K}$ (Ref. 13) might be related to a temperature dependence of the fluctuations. Furthermore, we did not find any evidence for a possible lower symmetry than Pmmn at room temperature, as it was proposed by Caimi et al. ${ }^{10,16}$ We failed to observe incommensurate superlattice reflections for the phase between $T_{c 2}$ and $T_{c 1}$, as was proposed in Ref. 13. Instead our results suggest a significant deviation of the structure from Pmmn between $T_{c 1}$ and $T_{c 2}$, that is replaced by a twofold superstructure below $T_{c 1}$, while an average Pmmn symmetry is restored for the basic structure. This scenario offers an explanation for the first-order character of the transition at $T_{c 1}$. The envisaged second-order spinPeierls transition from Pmmn towards the observed superstructure would have occurred at $T_{S P}<T_{c 2}$, but it is superseded by a structural transition at $T_{c 2}$ towards the intermediate phase with a distortion that is different from the dimerization in the spin-Peierls state. Below $T_{c 1}<T_{S P}$ the spin-Peierls state becomes the most stable state. It develops out of the intermediate phase, thus requiring major structural rearrangements, which explains the first-order character of the phase transition at $T_{c 1}$. Band-structure calculations have found that the electronic ground state for the roomtemperature structure is characterized by a $d_{x y}^{1}$ configuration of the $\mathrm{Ti}^{3+}$ ion. ${ }^{12}$ Accordingly it should also be the ground state at low temperatures. The transitions at $T_{c 1}$ then would lead to a further stabilization of the $d_{x y}$ orbital, and the suppression of fluctuations involving other $d$ orbitals, but it would not correspond to orbital order.

In conclusion, we have found that $\mathrm{TiOCl}$ exhibits a firstorder phase transition at $T_{c 1}=66(1) \mathrm{K}$ towards a superstructure with a doubled lattice constant $\mathbf{b}$ along the chains of $\mathrm{Ti}$ atoms and with monoclinic symmetry $P 2_{1} / m$, while at $T_{c 2}=90(1) \mathrm{K}$ the lattice symmetry has already become lower than orthorhombic. The superstructure is characterized by a simple dimerization of the chains of Ti atoms, while the displacements of the $\mathrm{O}$ and $\mathrm{Cl}$ atoms obtain values to minimize the internal strain of the superstructure. Despite the unconventional magnetic behavior observed at higher temperatures, our results provide compelling evidence for a spinPeierls state of TiOCl below $T_{c 1}=66(1) \mathrm{K}$, that is achieved through direct exchange between electron spins on the chains of Ti atoms parallel $\mathbf{b}^{7}$ The superstructure of $\mathrm{TiOCl}$ is surprisingly simple, much simpler than that of $\mathrm{CuGeO}_{3}$ (Ref. 5) and $\mathrm{TiOCl}$ can thus be identified as a spin-Peierls system with the relevant magnetic interactions confined to a true 1D chain of atoms and with the highest transition temperature yet observed.

We gratefully acknowledge support with the synchrotron experiment by W. Morgenroth. X-ray diffraction with synchrotron radiation was performed at beamline D3 of Hasylab at DESY (Hamburg, Germany). This work was supported by the German Science Foundation (DFG) through Grants No. SM55/6-3, CL124/3-3, and SFB484.
*Electronic address: smash@uni-bayreuth.de (URL: http://www. uni-bayreuth.de/departments/crystal/).

${ }^{1}$ R. J. Beynon and J. A. Wilson, J. Phys.: Condens. Matter 5, 1983 (1993).

${ }^{2}$ M. Hase, I. Terasaki, and K. Uchinokura, Phys. Rev. Lett. 70, 3651 (1993).

${ }^{3}$ J. P. Pouget, L. P. Regnault, M. Ain, B. Hennion, J. P. Renard, P. Veillet, G. Dhalenne, and A. Revcolevschi, Phys. Rev. Lett. 72, 4037 (1994).

${ }^{4}$ O. Kamimura, M. Terauchi, M. Tanaka, O. Fujita, and J. Akimitsu, J. Phys. Soc. Jpn. 63, 2467 (1994).

${ }^{5}$ K. Hirota, D. E. Cox, J. E. Lorenzo, G. Shirane, J. M. Tranquada, M. Hase, K. Uchinokura, H. Kojima, Y. Shibuya, and I. Tanaka, Phys. Rev. Lett. 73, 736 (1994).

${ }^{6}$ P. Lemmens, G. Güntherodt, and C. Gros, Phys. Rep. 375, 1 (2003).

${ }^{7}$ A. Seidel, C. A. Marianetti, F. C. Chou, G. Ceder, and P. A. Lee, Phys. Rev. B 67, 020405(R) (2003).

${ }^{8}$ H. Schäfer, F. Wartenpfuhl, and E. Weise, Z. Anorg. Allg. Chem. 295, 268 (1958).

${ }^{9}$ V. Kataev, J. Baier, A. Moller, L. Jongen, G. Meyer, and A.
Freimuth, Phys. Rev. B 68, 140405(R) (2003).

${ }^{10}$ G. Caimi, L. Degiorgi, N. N. Kovaleva, P. Lemmens, and F. C. Chou, Phys. Rev. B 69, 125108 (2004).

${ }^{11}$ M. Hoinkis et al. (unpublished).

${ }^{12}$ T. Saha-Dasgupta, R. Valenti, H. Rosner, and C. Gros, Europhys. Lett. 67, 63 (2004).

${ }^{13}$ T. Imai and F. C. Chou, cond-mat/0301425 (unpublished).

${ }^{14}$ P. M. de Wolff, T. Janssen, and A. Janner, Acta Crystallogr., Sect. A: Cryst. Phys., Diffr., Theor. Gen. Crystallogr. A37, 625 (1981).

${ }^{15}$ S. van Smaalen, Crystallogr. Rev. 4, 79 (1995).

${ }^{16}$ G. Caimi, L. Degiorgi, P. Lemmens, and F. C. Chou, J. Phys.: Condens. Matter 16, 5583 (2004).

${ }^{17}$ Seven hundred and fifty-eight (758) measured reflections reduce to 252 observed unique main reflections and 153 observed unique superlattice reflections in monoclinic symmetry. The refinement converged to a model with reliability factors $R_{\text {all }}=0.055$ with $R_{\text {main }}=0.051$ and $R_{\text {sat }}=0.083$. In orthorhombic symmetry 225 unique main reflections and 130 unique satellites were obtained. The refinement converged at $R_{\text {all }}=0.058$ with $R_{\text {main }}=0.055$ and $R_{\text {sat }}=0.081$. 